\title{
Influencia del Angulo de Inclinación de los Tubos Solares Evacuados sobre la Temperatura del Agua
}

\author{
Celso Recalde ${ }^{(1)}$, Cesar Cisneros $^{(2)}$, Carlos Ávila(3) $^{(3)}$ Gladys Urquizo(1) $^{(1)}$ \\ (1) Escuela Superior Politécnica de Chimborazo, Facultad de Ciencias, Panamericana Sur Km 11/2, \\ Riobamba-Ecuador (e-mail: crecalde672000@yahoo.com) \\ (2) Instituto Superior Politécnico José Antonio Echeverría, Centro de Estudios de Tecnologías Energéticas \\ Renovables, Habana-Cuba (e-mail: cesar@ceter.cujae.edu.cu) \\ (3) Escuela Politécnica Nacional, Ladrón de Guevara E11-253, Quito- Ecuador. \\ (e-mail: cavila67@yahoo.com).
}

Recibido Nov. 5, 2014; Aceptado Ene. 8, 2015; Versión final Abr. 21, 2015, Publicado Ago. 2015

\section{Resumen}

Se analizó el comportamiento de tubos evacuados ante la acción de la radiación solar incidente en la zona ecuatorial alto andina. Las pruebas se realizaron en cuatro días diferentes con tres declinaciones solares, cubriendo la época seca y la época lluviosa. Se trabajó con dos tubos evacuados, inclinados a 15으 y $60^{\circ}$; $20^{\circ}$ y $45^{\circ} ; 30^{\circ}$ y $60^{\circ} ; 10^{\circ}$ y $50^{\circ}$. Las mediciones demuestran que en las primeras horas del monitoreo la temperatura más alta del agua corresponde al tubo evacuado con el ángulo mayor y al acercarse el medio día el comportamiento cambia, a la inversa. Se concluye que para optimizar la incidencia de la radiación solar directa en el calentamiento de agua en la región ecuatorial se debe emplear tubos evacuados horizontales.

\section{Effect of Tilt Angle of Evacuated Tubes on the Temperature of the Water}

\begin{abstract}
The behavior of solar evacuated tubes under the action of solar radiation in the Equator Andean high lands is presented. The tests were performed during four different days with three solar declinations, covering the dry season and the rainy season. In this study evacuated tubes at inclinations $15^{\circ}$ and $60^{\circ} ; 20^{\circ}$ and $45^{\circ} ; 30^{\circ}$ and $60^{\circ} ; 10^{\circ}$ and $50^{\circ}$ were employed. The collected data shows that at the beginning of the monitoring experiment the highest temperatures was found for larger inclination angles. At noon, the behavior changes, to the inverse. It is concluded that solar evacuated tubes in the Equator Andean high lands must be in horizontal position to optimize the use of direct solar radiation.
\end{abstract}




\section{INTRODUCCIÓN}

La crisis energética por el uso prolongado de fuentes fósiles principalmente en la generación de electricidad, influye negativamente en el calentamiento global, en la degradación de la capa de ozono, con la presencia de lluvias ácidas que traen como consecuencia la degradación del ambiente y la calidad de vida (Yadav, 2011). Los colectores solares transforman la radiación solar en energía térmica, reemplazan el uso de electricidad y reducen la emisión de gases efecto invernadero. Además, los costos de operación y funcionamiento son muy bajos respecto a los dispositivos eléctricos y son amigables con el ambiente. Sin embargo, la inversión inicial es muy alta, trabajan solamente con radiación total (directa y difusa) y no funcionan adecuadamente en días nublados. Por otro lado, hay numerosos tipos de colectores para aplicaciones prácticas: de placa plana, de tubos térmicos y colectores de tubos evacuados o tubos al vacío, estos últimos se agrupan según su diseño en "fluido en metal" o "fluido en vidrio" (Azad, 2011). Los colectores de agua en vidrio están en el mercado por casi tres décadas, sin embargo, no fueron competencia real para los colectores de placa plana, sino hasta hace poco, al haber alcanzado alta eficiencia térmica con diseños simples y principalmente a bajo costo. Los dispositivos con tubos evacuados tienen un mejor comportamiento para calentar agua que los de placa plana (Ayompe, 2011), siendo la mejor opción para uso doméstico por su simplicidad y costo. En los últimos años se ha invertido en investigación y desarrollo de estos sistemas de calentamiento, planteándose modelos de radiación para colectores al vacío con absorbedor tubular.

Este proceso de investigación llevó al desarrollo de un método que determina el tamaño y posición de la sombra sobre el área de cada tubo (Duffie y Beckman, 2013), se ha monitoreado el comportamiento típico del agua al interior de los tubos evacuados y en el precalentamiento (Budihardjo et. al., 2007). Varios modelos han permitido entender los procesos de transferencia de calor y flujo de fluidos al interior de tubos con un extremo abierto (Khanafer y Vafai, 2000). Se han conducido programas experimentales que han permitido obtener correlaciones numéricas para el flujo de circulación natural a través de tubos evacuados de vidrio con un extremo abierto y montados sobre un reflector difuso (Budihardjo et. al., 2007). Sin embargo, hay pocas publicaciones sobre el comportamiento térmico de tubos evacuados para calentar agua con diferentes ángulos (Tang et. al., 2011), que correlacionen el caudal de circulación a través de los tubos, la influencia de la geofísica del lugar, el ingreso de calor, la temperatura del fluido (Selvakumar y Somasundaram, 2011), la geometría del tubo, las condiciones de calentamiento alrededor de las paredes del tubo (Saleh y Hasan, 2013), se han limitado únicamente a isotermas o condiciones de isoflujos. Por otro lado, el mercado de la zona ecuatorial es pequeño, con pocos trabajos de investigación y desarrollo.

En latitudes cercanas a la zona ecuatorial $25^{\circ}$ de latitud norte, (Tang et. al., 2011) concluye que para maximizar la ganancia anual de calor en los calentadores solares de agua, el colector debe ser inclinado a un ángulo que maximice la captación de la radiación solar a lo largo del año. Además, experimentos mostraron (Tang et. al., 2011) que tubos solares orientados sur norte con un ángulo de inclinación respecto a la horizontal, una parte del agua fría del tanque que baja hacia el extremo sellado puede regresar directamente al tanque sin haber alcanzado nunca el extremo sellado del tubo, esta fracción de agua se incrementa al aumentar el ángulo de inclinación. En la India, Selvakumar y Somasundaram (2011) estudió el comportamiento térmico de tubos evacuados para calentar agua con diferentes ángulos, sin encontrar variaciones significativas en la eficiencia térmica diaria, sin embargo, en la mayoría de los manuales se menciona que el ángulo de instalación óptimo de los tubos está entre: latitud-10ํy latitud+10․․

Los dispositivos termosolares con tubos evacuados que se instalan en Ecuador, poseen ángulos de inclinación en la mayoria de los casos entre $60^{\circ}$ y $40^{\circ}$, influenciados por la configuración del lugar donde fueron construidos y obtuvieron las certificaciones. Además, muchos profesionales en el tema solar persisten en instalar los dispositivos termosolares con ángulos de inclinación mayores a 40으 (Tang et. al., 2011), considerando que los colectores con inclinaciones inferiores, incrementan la porción del volumen de agua fría bajo el nivel de la abertura del tubo evacuado y reduce la porción solar en el tanque de agua. Sin embargo, se ha demostrado experimentalmente que estos efectos son despreciables en el funcionamiento de los dispositivos termosolares (Tang et. al., 2011).

La sierra alto andina presenta bajas temperaturas ambientales, especialmente en las horas de la noche, por estar en la zona tropical sus estaciones son poco marcadas (Recalde, et al. 2010). Con la mejora del nivel de vida, se ha incrementado el uso de dispositivos eléctricos, a gas y pequeñas estufas que calientan el ambiente con calor generado a partir de la combustión de leña y desperdicios agrícolas; afectando el clima, la agricultura y causando problemas respiratorios. Para calentar el agua sanitaria se emplea electricidad, gas, leña y dispositivos termosolares con diseños y especificaciones técnicas validadas bajo condiciones geofísicas diferentes; el comportamiento termodinámico al interior de los tubos evacuados en Ecuador se diferencia de otras latitudes principalmente por la magnitud de la aceleración de la gravedad $9,77 \mathrm{~m} / \mathrm{s}^{2}$ inferior a los $9,81 \mathrm{~m} / \mathrm{s}^{2}$ al nivel del mar; la presión atmosférica de aproximadamente 786 milibares inferior a 
los 1013 milibares a nivel del mar; la dirección del campo magnético terrestre y la fuerza de Coriolis que es horizontal en Ecuador, y finalmente se realizó esta investigación a 2853 m.s.n.m., hemisferio sur, zona 17M 9816967,05Y 758398,29X.

La duración y la cantidad de radiación solar dependen, por tanto, de la combinación de efectos astronómicos y meteorológicos. El potencial energético solar tiene una fuerte influencia de parámetros como la altura, el ángulo de inclinación, la orientación del dispositivo de captación y principalmente de las características atmosféricas y climatológicas reinantes. La revisión bibliográfica lleva al planteamiento de la siguiente pregunta fundamental que guía este trabjo de investigación: ¿Cuál es la insidencia de la inclinación de tubos evacuados en el comportamiento del fluido que colecta energía solar bajo las condiciones impuestas por una zona alto andina ecuatorial?. Para dar respuesta a esta pregunta de investigación, en este trabajo se monitoreó el comportamiento térmico del agua al interior de tubos evacuados, respecto al tiempo. Se realizaron cuatro pruebas en días diferentes, con tres declinaciones solares concluyéndose que en la zona ecuatorial alto andina, los ángulos pequeños respecto a la horizontal mejora la eficiencia del efecto termosifón y por tanto la transformación de la radiación solar en calor al interior de los tubos evacuados.

\section{METODOLOGÍA}

Para suplir la falta de piranómetros a diferentes ángulos, se estimó el comportamiento de la radiación solar directa incidente sobre la superficie terrestre en función de la hora solar y de la distancia Tierra - Sol a lo largo del año utilizando Ecn. (1) con 0,01\% de precisión (Duffie y Beckman, 2013).

$G_{o n}=G_{s c}(1,000110+0,034221 \cos B+0,001280 \sin B+0,000719 \cos 2 B+0,000077 \sin 2 B)$

$B=(n+1) \frac{360}{365}$

Donde: $G_{o n}$ es la radiación solar extraterrestre incidente sobre el plano normal en el enésimo día del año en $\mathrm{W} / \mathrm{m}^{2}, \mathrm{G}_{\mathrm{sc}}$ define a la constante solar de $1366,8 \mathrm{~W} / \mathrm{m}^{2}$. La función adimensional B incorpora la variación respecto al día del año. Las perturbaciones de la rotación terrestre definida por la posición angular del Sol al mediodía solar respecto del plano del ecuador $\delta$ Ecn. (3) (Duffie y Beckman, 2013) con un error menor de 0,0035 grados.

$$
\begin{aligned}
& \delta=(180 / \pi)(0,006918-0,399912 \cos \mathrm{B}+0,070257 \sin \mathrm{B}-0,006758 \cos 2 \mathrm{~B} \\
& +0,000907 \sin 2 \mathrm{~B}-0,002697 \cos 3 \mathrm{~B}+0,00148 \sin 3 \mathrm{~B})
\end{aligned}
$$

La radiación solar incidente sobre superficies inclinadas respecto a la horizontal y al meridiano local son función del ángulo cenital $\theta_{z}$ de acuerdo al esquema que se muestra en la Figura 1. Esta figura muestra al ángulo de posición del Sol respecto a la normal al plano de incidencia, en términos de la declinación $\delta$, latitud $\phi$, ángulo horario $\omega$, ángulo de inclinación de la superficie $\beta$ y su orientación respecto al meridiano $\gamma$ (Duffie y Beckman, 2013).

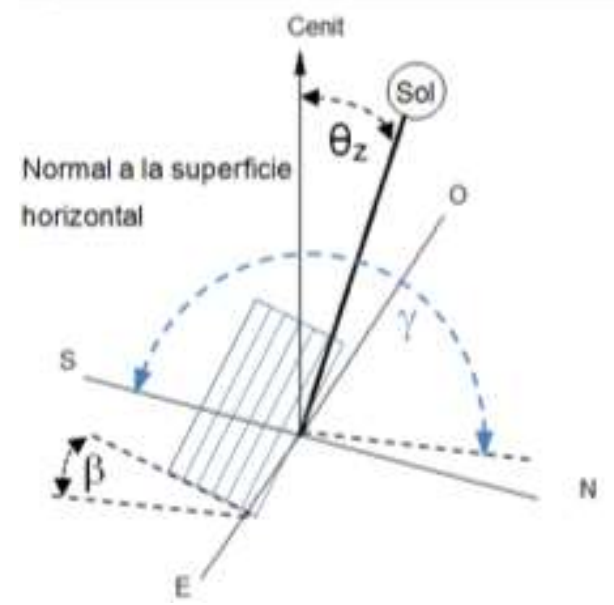

Fig. 1 Ángulo cenital $\theta_{z}$, ángulo de inclinación de la superficie $\beta$ y la orientación respecto al meridiano $\gamma$

$\cos \theta_{z}=\sin \delta \sin \Phi \cos \beta-\sin \delta \cos \Phi \sin \beta \cos \gamma+\cos \delta \cos \Phi \cos \beta \cos \omega+$

$\cos \delta \sin \Phi \sin \beta \cos \gamma \cos \omega+\cos \delta \sin \beta \sin \gamma \sin \omega$ 
Se estimó la incidencia de la radiación solar directa sobre planos inclinados utilizando la Ecn. 9 para la configuración mostrada en la Figura 2. Este modelo considera el efecto de la absorción y dispersión atmosférica en un cielo claro estándar, para altitudes inferiores a los $2,5 \mathrm{~km}$ en base a la Ecn. (5). El ángulo cenital $\theta_{z}$ se evalúa utilizando la Ecn. (4) (Duffie y Beckman, 2013). El modelo utiliza una altura $A=2853$ m.s.n.m. y por la posición geográfica de la zona de estudio se usó coeficientes de un clima tropical: $r_{0}=0,95$; $r_{1}=0,98 ; r_{k}=1,02$. Con $a_{0}$ evaluado con Ecn.(6), a determinado con la Ecn.(7) y k calculado con la Ecn.(8) se ajustan esto parámetros empíricamente de acuerdo a la propuesta de Samsón (2010), para atmósferas estándares con $5 \mathrm{~km}$ de visibilidad.

$$
\begin{aligned}
& \tau_{b}=a_{o}+a_{1} e^{\left(-k / \cos \theta_{z}\right)} \\
& a_{o}=r_{o}\left[0,4237-0,00821(6-A)^{2}\right] \\
& a_{1}=r_{1}\left[0,5055-0,00595(6,5-A)^{2}\right] \\
& k=r_{k}\left[0,2737-0,01858(2,5-A)^{2}\right] \\
& G_{b}=G_{o n} \cos \theta_{z} \tau_{b}
\end{aligned}
$$

La fase experimental de este estudio se inicia con la toma de valores de temperatura a partir de las $10 \mathrm{~h} 00$ para conseguir que la radiación solar directa incida sobre la parte superior de los tubos evacuados y teniendo en cuenta que en la mañana prevalece la radiación difusa. La configuración del experimento se muestra en la Figura 2.

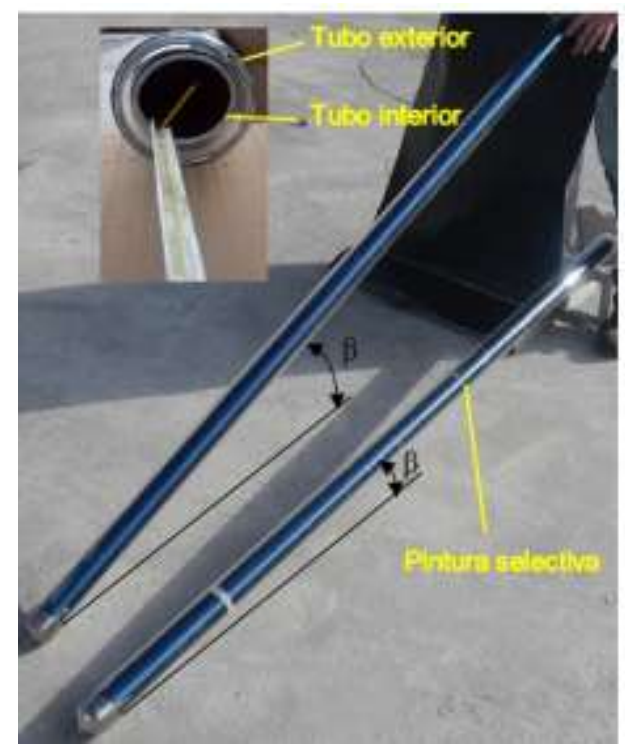

Fig. 2 Disposición de los tubos evacuados y en recuadro termocupla tipo K.

Los tubos evacuados consisten en dos cilindros de vidrio coaxiales entre los cuales se ha creado vacío, con un extremo abierto; el tubo exterior posee mínima reflexión y es transparente a los rayos de luz; el tubo interior es recubierto con pintura selectiva con alta absorción a la radiación y baja reflexión. La longitud de los colectores es de 1,8 $\mathrm{m}$ de estos $1,73 \mathrm{~m}$ con recubrimiento, el diámetro exterior del tubo evacuado es de $58 \mathrm{~mm}$, el diámetro interior de $42 \mathrm{~mm}$, el diámetro del tubo con recubrimiento es de $47 \mathrm{~mm}$, el espesor del vidrio exterior $1,87 \mathrm{~mm}$ y el espesor del vidrio interno incluido el recubrimiento es de 1,68 $\mathrm{mm}$. Se monitorea la temperatura en tres puntos a lo largo de cada uno de los dos tubos evacuados, dispuestos con distintos ángulos de inclinación respecto a la horizontal: $15^{\circ}$ y $60^{\circ} ; 20^{\circ}$ y $45^{\circ} ; 30^{\circ}$ y $60^{\circ} ; 10^{\circ}$ y $50^{\circ}$ en una configuración similar a la mostrada en la Figura 2 y ubicados en la terraza de un edificio de cuatro pisos.

Los dispositivos se orientaron con un ángulo $\gamma=160^{\circ}$ entre la proyección de la normal de la superficie sobre el plano horizontal y el meridiano local. Las cuatro pruebas se ralizaron el 28 y 29 de noviembre del 2013 temporada seca, el 11 de marzo y el 21 de mayo del 2014 temporada lluviosa. A pesar que en Ecuador no hay estaciones marcadas, hay más variación entre las temperaturas del día y la noche, que entre las temperaturas de las temporadas de lluvia y de seca. En los cuatro casos, el monitoreo concluyó al alcanzar el burbujeo previo a la ebullición, que se presentó aproximadamente a los $87^{\circ} \mathrm{C}$, ver Tabla 1.

Para la toma automática de datos se implementó un módulo electrónico de adquisición y almacenamiento que permitió trabajar con tres termocuplas tipo $\mathrm{K}$ previamente calibradas en cada tubo. La precisión de las 
termocuplas especificada es de $\pm 1,3^{\circ} \mathrm{C}$. Se midió la temperatura de cada tubo evacuado cerca del extremo abierto $T_{1}$, en la parte media $T_{2}$ y cerca del extremo sellado $T_{3}$. Los sensores de temperatura se conectaron a tarjetas Arduino uno y a un adquisidor de datos DAQ NI-6009 que además, procesa y transmite la información. Adicionalmente se utilizó una aplicación con interfaz gráfica para almacenar las temperaturas medidas en una base de datos. La intensidad de la radiación solar global sobre la horizontal en las dos pruebas realizadas el 2013 fue medida por un piranómetro solar 130 de una estación meteorológica automática Vaisalade de propiedad de la Universidad Nacional de Chimborazo, ubicada a una distancia de $4 \mathrm{Km}$. El monitoreo de la radiación solar global y difusa de marzo y mayo del 2014 se lo realizó con piranómetros de primera clase ISO 9060 de una estación meteorológica automática Vaisala de propiedad de la Escuela Superior Politécnica de Chimborazo y ubicada a 300 metros de donde se realizó el experimento. La Figura 6 muestra los datos medidos cada cinco minutos del 11 de marzo del 2014.

El calor colectado es función de la intensidad de la radicación solar incidente sobre los tubos. El fluido se calienta por conducción y principalmente por convección natural que activa la circulación termosifónica en los tubos evacuados. Los perfiles de temperatura fueron monitoreados a intervalos de tres minutos desde la mañana hasta inicios de la tarde en días con características de cielo claro. Para determinar la velocidad de calentamiento del agua se calculó las pendientes y los coeficientes de correlación de las temperaturas respecto al tiempo en las tres posiciones de cada tubo evacuado, los datos se muestran en la Tabla 1.

\section{RESULTADOS Y DISCUSIÓN}

El modelo matemático del camino solar genera resultados que muestran que en las primeras horas de la mañana del 28 y 29 de noviembre del 2013 y el 11 de marzo del 2014, la radiación solar directa incide de sur a norte en la parte inferior de los tubos. El monitoreo se inició a las 10h00, tan solo minutos antes de que la radiación solar directa incida sobre la parte superior del tubo evacuado inclinado 60․ Para inclinaciones menores de los tubos evacuados la incidencia de la radiación solar directa inicia mas temprano en la mañana, Figura 3.

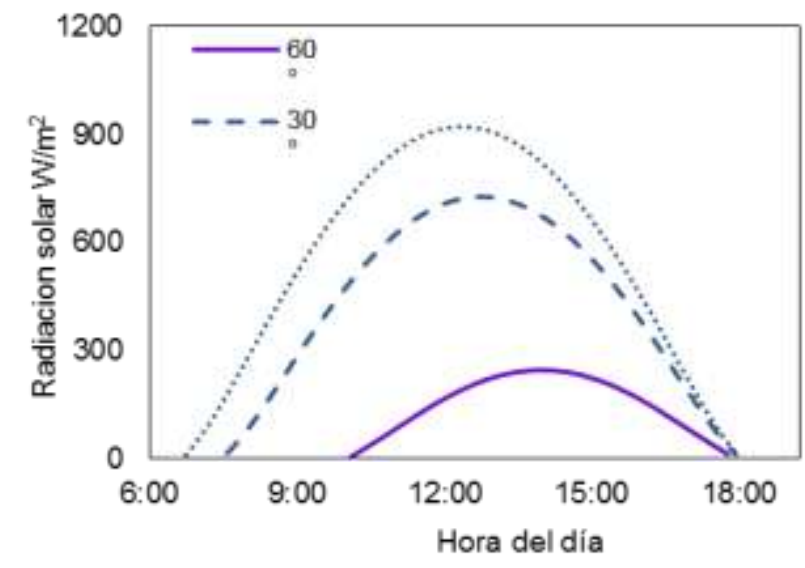

Fig. 3: Radiación solar incidente modelada a tres inclinaciones del tubo evacuado y $\delta=-21^{\circ}$.

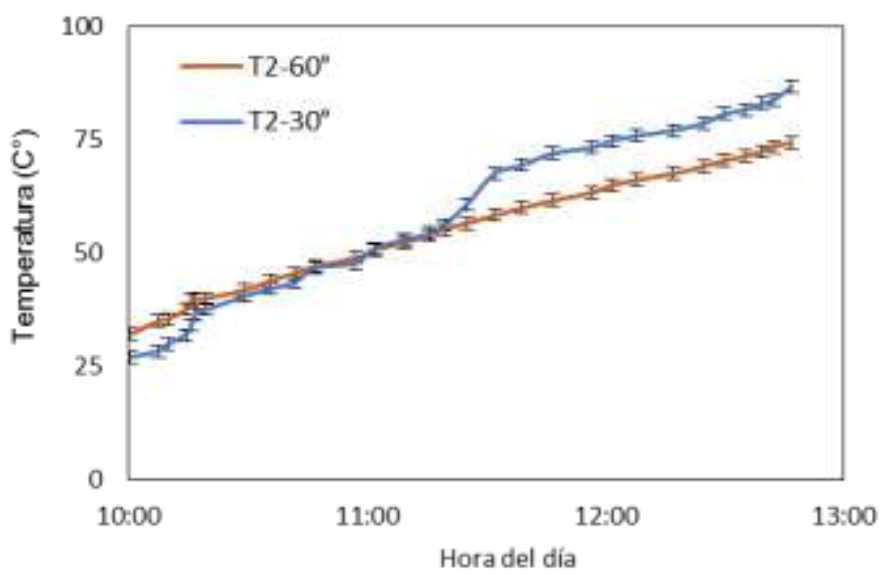

Fig. 4: Comportamiento de la temperatura respecto al tiempo primera prueba.

Durante la primera hora de monitoreo se observan valores menores de temperatura al interior del tubo inclinado a $30^{\circ}$ respecto al tubo inclinado $60^{\circ}$, ver Figura 4. Este fenómeno se explica pues la radiación solar 
directa inside de sur a norte en la parte inferior de los dispositivos, ver Figura 3. Este fenómeno se observa de mejor manera en días con baja nubosidad.

La prueba tres tuvo lugar el 11 de marzo del 2014 con poca presencia de nubosidad y una declinación solar de $-4^{\circ}$, el monitoreo se inició a las $11 \mathrm{~h} 00$. EL experimento muestra que los valores de temperatura del agua al interior de los tubos evacuados a $20^{\circ}$ de inclinación, en las tres posiciones, son superiores a los valores de las temperaturas en el tubo a $45^{\circ}$ de inclinación. Finalmente, el 21 de mayo del 2014, con una declinación solar positiva de $20^{\circ}$, se confirma el esta tendencia en el comportamiento de la temperatura del agua. El tiempo promedio de insolación de las cuatro pruebas fue de tres horas. Se calculó las pendientes de las curvas temperatura vs tiempo, mostrándo en todos los casos que los valores mayores de la velocidad con que crece la temperatura del agua corresponde a los tubos evacuados con ángulos menores, estos datos se muestran en la Tabla 1. Los efectos de flotabilidad por diferencias de densidades del agua provocan que los valores de la temperatura $T_{1}$ correspondiente a la parte superior del tubo evacuado, presente los valores mayores en todas las pruebas. Por otro lado la temperatura $T_{3}$ cerca del extremo sellado muestra un comportamiento en función al tiempo con menor crecimiento, ver Fig. 5 y Tabla 1.

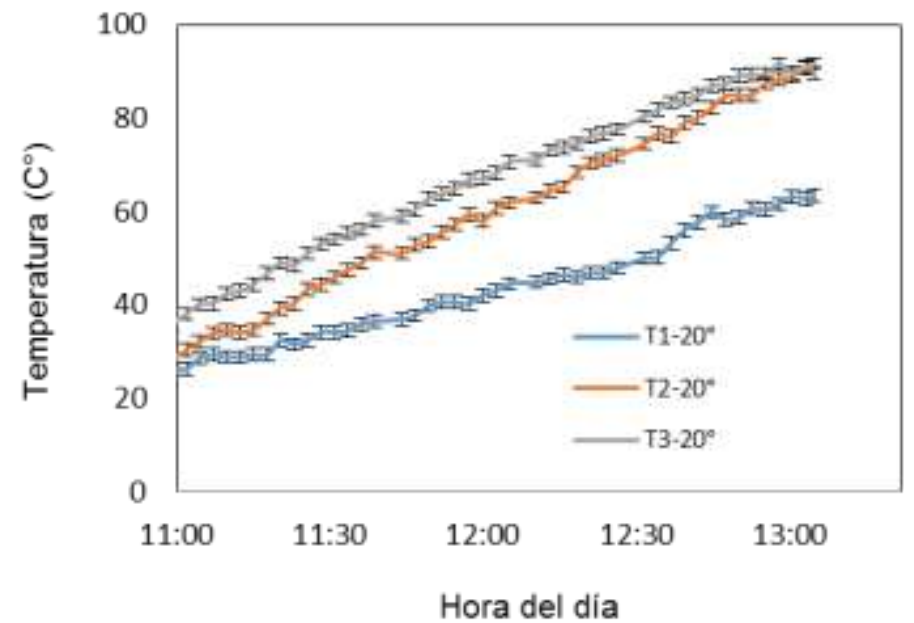

Fig. 5: Comportamiento del perfil de temperatura al interior del tubo a $20^{\circ}$ de inclinación

Tabla. 1: Comparación datos experimentales de los cuatro casos

\begin{tabular}{|c|c|c|c|c|c|c|c|c|}
\hline Fecha & \multicolumn{2}{|c|}{$28 / 11 / 13$} & \multicolumn{2}{|c|}{$29 / 11 / 13$} & \multicolumn{2}{|c|}{$11 / 03 / 14$} & \multicolumn{2}{|c|}{$21 / 05 / 14$} \\
\hline Hora de inicio & \multicolumn{2}{|c|}{$10 \mathrm{~h} 00$} & \multicolumn{2}{|c|}{ 10h03 } & \multicolumn{2}{|c|}{ 10h54 } & \multicolumn{2}{|c|}{$11 \mathrm{~h} 01$} \\
\hline$\delta(\stackrel{\circ}{)})$ & \multicolumn{2}{|c|}{$-21,2$} & \multicolumn{2}{|c|}{$-21,4$} & \multicolumn{2}{|c|}{-4} & \multicolumn{2}{|c|}{20} \\
\hline Tiempo monitoreo (h) & \multicolumn{2}{|c|}{2,8} & \multicolumn{2}{|c|}{4,7} & \multicolumn{2}{|c|}{2,3} & \multicolumn{2}{|c|}{2,0} \\
\hline Radiación solar global W/m² & \multicolumn{2}{|c|}{626} & \multicolumn{2}{|c|}{602} & \multicolumn{2}{|c|}{1047} & \multicolumn{2}{|c|}{1003} \\
\hline Temperatura ambiente $\left({ }^{\circ} \mathrm{C}\right)$ & \multicolumn{2}{|c|}{15} & \multicolumn{2}{|c|}{14,6} & \multicolumn{2}{|c|}{13,7} & \multicolumn{2}{|c|}{14,3} \\
\hline Presión atmosférica (mmHg) & \multicolumn{2}{|c|}{547} & \multicolumn{2}{|c|}{547} & \multicolumn{2}{|c|}{548} & \multicolumn{2}{|c|}{549} \\
\hline Inclinación tubo ( $\stackrel{\circ}{*})$ & 60 & 30 & 60 & 15 & 45 & 20 & 50 & 10 \\
\hline Temperatura inicial $\mathrm{T} 1\left({ }^{\circ} \mathrm{C}\right)$ & 37 & 35 & 23 & 24 & 32 & 29 & 44 & 39 \\
\hline Temperatura final $\mathrm{T} 1\left({ }^{\circ} \mathrm{C}\right)$ & 76 & 88 & 93 & 93 & 88 & 91 & 86 & 91 \\
\hline Pendiente $\mathrm{T}_{1}$ & 1,61 & 2,42 & 1,73 & 1,91 & 3,11 & 3,39 & 2,75 & 3,40 \\
\hline Pendiente $\mathrm{T}_{2}$ & 1,81 & 2,67 & 1,96 & 2,12 & 3,36 & 3,74 & 3,27 & 3,70 \\
\hline Pendiente $\mathrm{T}_{3}$ & 1,19 & 1,94 & 1,55 & 2,22 & 2,20 & 2,22 & 1,95 & 2,16 \\
\hline
\end{tabular}

Esta tendencia muestra que el efecto termosifón se da con mayor dificultad en el fluido cerca al extremo sellado. La viscosidad y la densidad disminuyen a mayores temperaturas mejorando la eficiencia del efecto termosifón. Debido al movimiento de rotación terrestre y a la trayectoria del sol en la zona ecuatorial al medio día, la intensidad de radiación es mayor, ver Figura 6 y además se aprovecha una mayor área de captación. Como consecuencia los tubos evacuados a ángulos pequeños cercanos a cero grados captan mayor cantidad de energía, como se evidencia en los valores de temperatura del agua mostrados en la Tabla 1. El burbujeo primero se evidenció en los tubos evacuados con inclinaciones menores. En la etapa final del monitoreo, se incrementó las pérdidas de calor debido a las mayores diferencias de temperaturas 
en el interface agua aire atmosférico. En esta etapa del experimento se presentó el cambio de fase líquido a gas, lo cual se refleja en el comportamiento de la temperatura al pasar de una tendencia lineal ascendente a una curva asintótica horizontal alrededor de los $92^{\circ} \mathrm{C}$, ver Fig. 5.

El coeficiente de determinación de la regresión lineal de los perfiles de temperatura respecto al tiempo en todos los casos es mayor cuando los tubos evacuados tienen ángulos de inclinación más altos, debido a que la radiación directa tiene un comportamiento más inestable respecto a la radiación difusa. En las pruebas se observó que el incremento del ángulo de inclinación suaviza la variabilidad de la radiación solar directa absorbida por el tubo evacuado tal como se ve en las Fig. 4 y 6.

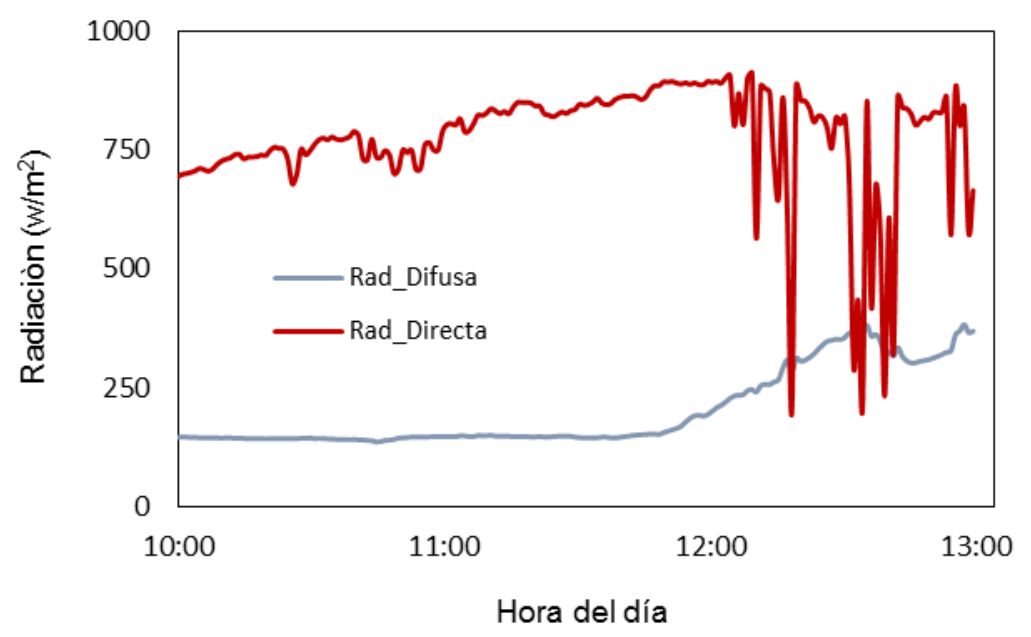

Fig. 6: Radiación directa y difusa el 11 de marzo 2014.

\section{CONCLUSIONES}

La superficie de incidencia de la radiación solar sobre los tubos evacuados tiene dependencia del ángulo de incidencia de la radiación solar directa, observándose que la temperatura del agua en los dispositivos a inclinaciones menores cambia en función de la radiación directa. Para días con alta presencia de nubosidad el ángulo de incidencia es menos significativo en la recolección de la radiación solar diaria.

El comportamiento diferente del perfil de temperatura $T_{3}$ cerca del extremo sellado muestra que existe una zona de menor influencia del efecto termosifón en la parte inferior del tubo evacuado.

Los resultados experimentales con diferentes inclinaciones de los tubos evacuados con tres declinaciones solares diferentes, muestra la influencia significativa en la recolección de la radiación solar diaria y por tanto en la captación de calor del sistema. El ángulo de instalación respecto a al horizontal de dispositivos con tubos al vacío en la zona ecuatorial andina es de $0^{\circ}$.

\section{AGRADECIMIENTOS}

Los autores agradecen a la SENESCYT por crear las condiciones para desarrollar ciencia en el Ecuador, al INER por la confianza en el Grupo Energías Alternativas y Ambiente de la ESPOCH y a la fundación italiana CDF por su permanente apoyo.

\section{REFERENCIAS}

Ayompe, L. y otros cuatro autores, Comparative field performance study of flat plate and heat pipe evacuated tube collector (ETCs) for domestic water heating systems in a temperate climate. DOI:10.1016/j.energy.2011.03.034; Energy, 36(5), 3370-3378 (2011)

Azad, E., Theoretical analysis to investigate thermal performance of co-axial heat pipe solar collector. DOI: 10.1007/s00231-011-0827-3; Heat and Mass Transfer, 47(12), 1651-1658 (2011)

Budihardjo, I., Morrison, G. y Behnia, M., Natural Circulation Flow through Water-in-Glass Evacuated Tube Solar Collectors. Solar Energy, 81(12), 1460-1472. (2007)

Duffie, J., Beckman, W., Solar Engineering of Thermal Processes, Fourth Edition, 2-133, John Wiley \& Sons, Inc. New Jersey, USA (2013) 
Goswami, Y. y Zhao, Y., Performance Model for Water-in-Glass Evacuated Tube Solar Water Heaters. DOI: 10.1007/978-3-540-75997-3_410; Solar Energy and Human Settlement, 1, 2018-2022. (2009)

Gray, L. y otros doce autores, Solar influences on climate, DOI:10.1029/2009RG000282; Rev. Geophys., 48, $1-53(2010)$

Igbal, M., An Introduction to Solar Radiation. 1st Edition, vol 1, 1-34, Academic Press Inc., New York, USA, (1983).

Khanafer, K. y Vafai, K., Buoyancy-Driven Flow and Head Transfer in Open-Ended enclosures: elimination of the extended boundaries. ISSN 0017-9310 (en línea); Int. Journal of Heat and Mass Transfer, 43(22), 40874100. (2000)

Machado, A. y otros tres autores. Estudio del Secado de Anacardo (Anacardium occidentale L.) mediante Secador Solar de Radiación Directa. DOI: 10.4067/S0718-07642010000100006; Información Tecnológica, 21(1), 31-37 (2010)

Monne, C., Alonso, S., Palacin, F., Evaluación de una Instalación de Refrigeración por Absorción con Energía Solar. DOI: 10.4067/S0718-07642010000100006; Información Tecnológica, 22(3), 39-44 (2011)

Lara, F. y otros tres autores. Metodología para el Dimensionamiento y Optimización de un Concentrador Lineal Fresnel. DOI: 10.4067/S0718-07642013000100013; Información Tecnológica, 24(1), 115-128 (2013)

Lema, A. y otros tres autores. Análisis del Efecto de Variables de Diseño en el Comportamiento de una Secadora de Granos de Amaranto. DOI: 10.4067/S0718-07642005000500008; Información Tecnológica, 6(5), 43-48 (2005)

Peixóto, J., Oort, A., Physics of climate. DOI 10.1103/RevModPhys; Rev. Mod. Phys. 56, 365-430 (1984)

Peña, D., Análisis de datos multivariantes. Primera edición, vol 24, Editorial McGraw-Hill. Madrid, España, (2002)

Recalde, C.y otros cinco autores. Los Tesoros del Qhapaq Ñan. Primera edición, vol 1, 85-112,Editorial el Conejo. Riobamba, Ecuador, (2010)

Saleh, S y Hasan, A., Evaluation of Convective Heat Transfer and Natural Circulation in an Evacuated Tube Solar Collector. ISSN 17264073 (en línea); Journal of Engineering. 5(19), 613-628. (2013)

Selvakumar, P y Somasundaram, P., Effect of Inclination Angle on Temperature Characteristics of Water inGlass Evacuated Tubes of Domestic Solar Water Heater. Int. Journal of Engineering and Innovative Technology. 1(4), 78-81 (2012)

Tang, R., Yang, Y. y Gao, W., Comparative studies on thermal performance of water-in-glass evacuated tube solar water heaters with different collector tilt-angles. DOI:10.1016/j.solener.2011.03.019; Solar Energy. 85(7), 1381-1389 (2011)

Villeda, G. y otros tres autores. Seguidor Solar de Dos Ejes para un Horno Solar. DOI: 10.4067/S071807642011000200011; Información Tecnológica, 22(2), 95-106 (2011)

Yadav, A. y Bajpai, V., An experimental study on evacuated tube solar collector for heating of air in India. Proceedings of World Academy of Science, ISNN 1307-6892 (en linea), http://waset.org/Publications? $p=55$. Acceso 5 mayo del 2014; Engineering and Technology, 5(7), 44-49 (2011) 